\title{
THE SELBERG TRACE FORMULA. V: QUESTIONS OF TRACE CLASS
}

\author{
BY \\ M. SCOTT OSBORNE AND GARTH WARNER ${ }^{1}$
}

\begin{abstract}
The purpose of this paper is to develop criteria which will ensure that the $K$-finite elements of $C_{c}^{\infty}(G)$ are represented on $L_{\mathrm{dis}}^{2}(G / \Gamma)$ by trace class operators.
\end{abstract}

1. Introduction. This is the fifth in a projected series of papers in which we plan to come to grips with the Selberg trace formula, the ultimate objective being a reasonably explicit expression. Let $G$, therefore, be a reductive Lie group and $\Gamma$ a lattice in $G$, both subject to the usual conditions. Consider, for the moment, the special case when $\operatorname{rank}(\Gamma)=1$. Given a $K$-type $\delta$, suppose that the poles of the $c$-functions $\mathbf{c}_{\delta, 0}$ in the interval $[-|\rho|, 0 \mid$ are bounded uniformly away from 0 in the orbit types 0 . Then

$$
\forall \alpha \in C_{c}^{\infty}(G) \text { st } \bar{\chi}_{\delta} * \alpha=\alpha,
$$

the operator $L_{G / \Gamma}^{\text {dis }}(\alpha)$ is trace class. In the present paper, this assertion will be established. Actually, its proof turns out to be quite easy, so our primary goal will be to formulate and prove the requisite generalization for $\Gamma$ of any rank, a task offering additional difficulties. During our consideration of this problem, we discovered a weaker condition, sufficient, nevertheless, to allow one to draw the same conclusion, the point being that the poles in the rank-one case, say, can be allowed to approach the origin provided they do so in a "tempered" way.

The issue of trace class on the full discrete spectrum is a vexing question of longtime standing. In this connection, recall that

$$
L_{\text {dis }}^{2}(G / \Gamma)=L_{\text {cus }}^{2}(G / \Gamma) \oplus L_{\text {res }}^{2}(G / \Gamma) .
$$

Now, on the cuspidal spectrum, the relevant operators are, as is well known, trace class. Consequently, it is the residual spectrum where all the problems lie. The conditions behind our hypotheses serve to guarantee trace class, but, of course, their validity has yet to be shown (except in familiar, elementary situations).

Various preliminaries of a general nature are dealt with in $\S 2$, while $\S 3$ is devoted to a compilation of certain facts about the eigenfunctions in $L_{\text {res }}^{2}(G / \Gamma)$. The residue hypothesis (and its generalization) is formulated in $\S 4$, where there is also to be found an ad-hoc discussion when $\operatorname{rank}(\Gamma)=1$. The proofs that the hypotheses lead to trace class are given in $\S 7$ and $\S 8$, the demonstrations depending on an important uniformity possessed by the degrees of the constant term polynomials (obtained in $\S 5$ ), in conjunction with the Laplace transform theory of $\S 6$. Certain

Received by the editors May 10, 1983 and, in revised form, December 2, 1983.

1980 Mathematics Subject Classification. Primary 10D40, 32N10; Secondary 22E40, 22 E46.

${ }^{1}$ Research of both authors supported in part by the National Science Foundation 
simple consequences are noted in $\S 9$. We terminate in $\S 10$ with a series of miscellaneous remarks; although they have been placed at the end, one may view them as providing additional orientation.

As a general reference and suggested overall introduction to the subject, we shall use our monograph The theory of Eisenstein systems, Academic Press, New York, 1981. Throughout the sequel, the title of this work has been abbreviated to TES.

Finally, we would like to thank the referee for several suggestions which have helped to clarify the presentation of the material,

2. Preliminaries. The purpose of this section is to establish a criterion of a general character, the thrust of which is to ensure that certain operators are of the trace class. This desideratum will then be applied to the analysis of the discrete spectrum in $L^{2}(G / \Gamma)$.

Initially, let us agree to place ourselves in the setting of the Appendix to Chapter 8 in TES. So let $G$ be a Lie group and $K$ a compact subgroup of $G$. Given a finite subset $F$ of $\hat{K}$, assign to $\chi_{F}$ and $\bar{\chi}_{F}$ their usual meanings (especially when $F=\{\delta\}$, say).

Generically, $U$ will stand for a unitary representation of $G$ on a Hilbert space $H_{U}$.

LEMMA 2.1. Fix $F \subset \hat{K}$. Then the following are equivalent:

(1) $\forall \alpha \in C_{c}^{\infty}(G) s t$

$$
\bar{\chi}_{F} * \alpha=\alpha \text { and } \alpha * \bar{\chi}_{F}=\alpha,
$$

$U(\alpha)$ is Hilbert-Schmidt.

(2) $\forall \alpha \in C_{c}^{\infty}(G) s t$

$$
\bar{\chi}_{F} * \alpha=\alpha \quad \text { or } \quad \alpha * \bar{\chi}_{F}=\alpha
$$

$U(\alpha)$ is Hilbert-Schmidt.

(3) $\exists p>0$ st $\forall \alpha \in C_{c}^{p}(G)$ st

$$
\bar{\chi}_{F} * \alpha=\alpha \text { and } \alpha * \bar{\chi}_{F}=\alpha,
$$

$U(\alpha)$ is Hilbert-Schmidt.

(4) $\exists p>0$ st $\forall \alpha \in C_{c}^{p}(G)$ st

$$
\bar{\chi}_{F} * \alpha=\alpha \quad \text { or } \quad \alpha * \bar{\chi}_{F}=\alpha,
$$

$U(\alpha)$ is Hilbert-Schmidt.

(5) $\forall \alpha \in C_{c}^{\infty}(G)$ st

$$
\bar{\chi}_{F} * \alpha=\alpha \quad \text { and } \quad \alpha * \bar{\chi}_{F}=\alpha
$$

$U(\alpha)$ is trace class.

(6) $\forall \alpha \in C_{c}^{\infty}(G) s t$

$$
\bar{\chi}_{F} * \alpha=\alpha \quad \text { or } \quad \alpha * \bar{\chi}_{F}=\alpha,
$$

$U(\alpha)$ is trace class.

(7) $\exists p>0$ st $\forall \alpha \in C_{c}^{p}(G)$ st

$$
\bar{\chi}_{F} * \alpha=\alpha \quad \text { and } \quad \alpha * \bar{\chi}_{F}=\alpha,
$$

$U(\alpha)$ is trace class. 
(8) $\exists p>0$ st $\forall \alpha \in C_{c}^{p}(G)$ st

$$
\bar{\chi}_{F} * \alpha=\alpha \quad \text { or } \quad \alpha * \bar{\chi}_{F}=\alpha
$$

$U(\alpha)$ is trace class.

(The proof may be found in TES, pp. 375-376.)

Let $\mathfrak{G}$ be the universal enveloping algebra of the complexification of the Lie algebra $\mathfrak{g}$ of $G$. Fix a positive semidefinite, elliptic element $\Delta$ in $\mathfrak{G}$ which is, in addition, Hermitian and $K$-central. Then, by a well-known theorem of Nelson and Stinespring,

$$
U_{\infty}\left(1+\Delta^{n}\right) \quad(n \in \mathbf{N})
$$

is essentially selfadjoint. Moreover, the corresponding closure is injective on its domain, admitting an everywhere defined, bounded inverse.

(Note. Here, of course, $U_{\infty}$ is the canonical representation of $\mathfrak{G}$ on the space of differentiable vectors in $H_{U}$.)

THEOREM 2.2. Let $T: H_{U} \rightarrow H_{U}$ be a bounded linear transformation. Then, in order that there exist a $p>0$ with the property that

$$
\forall \alpha \in C_{c}^{p}(G) \text { st } \alpha * \bar{\chi}_{F}=\alpha,
$$

$U(\alpha) T$ is Hilbert-Schmidt, it is necessary and sufficient that there exist an $N>0$ with the property that

is Hilbert-Schmidt.

$$
{\overline{U_{\infty}\left(1+\Delta^{N}\right)}}^{-1} U\left(\bar{\chi}_{F}\right) T
$$

ProOF. Sufficiency. We have

$$
\begin{aligned}
U(\alpha) T & =U(\alpha) U\left(\bar{\chi}_{F}\right) T \\
& =U(\alpha) \overline{U_{\infty}\left(1+\Delta^{N}\right)} \cdot \overline{U_{\infty}\left(1+\Delta^{N}\right)}-1 \\
& =\left(\bar{\chi}_{F}\right) T \\
& =U\left(\alpha *\left(1+\Delta^{N}\right)\right) \cdot{\overline{U_{\infty}\left(1+\Delta^{N}\right)}}^{-1} U\left(\bar{\chi}_{F}\right) T,
\end{aligned}
$$

if $\alpha \in C_{c}^{p}(G)$, where $p \geq \operatorname{ord}(\Delta) \cdot \mathrm{N}$.

Necessity. On the basis of the theory of the parametrix (see, e.g., [16]), given $p>0$, there is an $N>0$ and $\mu \in C_{c}^{p}(G), \nu \in C_{c}^{\infty}(G)$ such that

$$
\Delta^{N} * \mu=\delta+\nu \quad\left(\delta=\text { Dirac measure at } 1_{\mathrm{G}}\right)
$$

in the sense of the theory of distributions on $G$. That being, by differentiable approximation and closedness,

$$
\operatorname{Ran}(U(\mu)) \subset \operatorname{Dom}\left(\overline{U_{\infty}\left(1+\Delta^{N}\right)}\right) .
$$

Write now $U(\mu)+U(\nu)$ as

$$
\overline{U_{\infty}\left(1+\Delta^{N}\right)} \cdot\left[U(\mu)-{\overline{U_{\infty}\left(1+\Delta^{N}\right)}}^{-1}\right] .
$$

Since trivially $U(\mu)+U(\nu)$ is also equal to

$$
\overline{U_{\infty}\left(1+\Delta^{N}\right)} \cdot\left[\overline{U_{\infty}\left(1+\Delta^{N}\right)}-1 U(\mu+\nu)\right] \text {, }
$$

bearing in mind that $\overline{U_{\infty}\left(1+\Delta^{N}\right)}$ is one-to-one, we can therefore say that

$$
U(\mu)-{\overline{U_{\infty}\left(1+\Delta^{N}\right)}}^{-1}={\overline{U_{\infty}\left(1+\Delta^{N}\right)}}^{-1} U(\mu+\nu) .
$$

But then

$$
{\overline{U_{\infty}\left(1+\Delta^{N}\right)}}^{-1} U\left(\bar{\chi}_{F}\right) T=U\left(\mu * \bar{\chi}_{F}\right) T-{\overline{U_{\infty}\left(1+\Delta^{N}\right)}}^{-1} \cdot U\left((\mu+\nu) * \bar{\chi}_{F}\right) T .
$$

Hence the theorem. 
COROLlaRY 2.3. $U(\alpha)$ is trace class for every $K$-finite $\alpha \in C_{c}^{\infty}(G)$ iff $\forall \delta \in \hat{K}$, $\exists N_{\delta}>0$ such that

$$
{\overline{U_{\infty}\left(1+\Delta^{N_{\delta}}\right)}}^{-1} U\left(\bar{\chi}_{\delta}\right)
$$

is Hilbert-Schmidt.

(In the preceding theorem, take for $T$ the identity map on $H_{U}$ and then quote Lemma 2.1.)

The above considerations may be applied to a reductive $G$ satisfying the usual conditions of Harish-Chandra, $K$ then being a maximal compact subgroup. As is customary in this situation, we shall specialize $\Delta$ to the positive semidefinite, elliptic element in $\mathfrak{G}$ canonically constructed from the Casimir $\omega: \Delta=\omega_{K}-\omega$, $\omega_{K} 2$ times the Casimir operator on $K$. Naturally, $\Delta$ is Hermitian and $K$-central.

Let $\Gamma$ be a lattice in $G$, ordinarily nonuniform, for which the basic assumption from TES is in force. Introduce the truncation operators $Q^{\mathbf{H}}(\mathbf{H} \in \mathfrak{a})$. Referring to [10] for the particulars, let us recall only that $\forall \mathbf{H} \in \mathfrak{a}_{Q}$,

$$
Q^{\mathbf{H}} \circ Q^{\mathbf{H}}=Q^{\mathbf{H}}, \quad\left(Q^{\mathbf{H}}\right)^{*}=Q^{\mathbf{H}} \quad \text { and } \quad \mathrm{Q}^{\mathbf{H}}\left(\mathrm{S}_{\mathrm{r}}^{\infty}(\mathrm{G} / \Gamma)\right) \subset \mathrm{R}(\mathrm{G} / \Gamma),
$$

$R(G / \Gamma)$ the space of rapidly decreasing functions on $G / \Gamma$.

Proposition 2.4. Let $\mathbf{H} \in \mathfrak{a}_{Q}$. Then $\forall \delta \in \hat{K}, \exists N_{\delta}>0$ such that

$$
Q^{\mathbf{H}} \circ \overline{\left(L_{G / \Gamma}\right)_{\infty}\left(1+\Delta^{N_{\delta}}\right)}{ }^{-1} L_{G / \Gamma}\left(\bar{\chi}_{\delta}\right)
$$

is Hilbert-Schmidt.

(One need only combine the criterion established in Theorem 2.2 with the proof of Proposition 4.5 of [9], the passage to arbitrary rank being evidently legitimate.)

3. Exponents of the eigenfunctions. The purpose of this section is to set the stage for the formulation of the residue hypothesis and its generalization $(\S 4)$ via a series of remarks on the eigenfunctions in the discrete spectrum of $L^{2}(G / \Gamma)$ and their exponents. Of course, the central focus will be on the residual spectrum, the issue of trace class on the cuspidal spectrum having been settled long ago by Gelfand and Pyatetskii-Shapiro.

The analysis of the spectral decomposition of $L^{2}(G / \Gamma)$ is the subject of Chapter 7 in TES, the notation of which we shall feel free to use without further comment. Thus, as there, we can write $L_{\mathrm{dis}}^{2}(G / \Gamma)$ in terms of an orthogonal direct sum over the association classes $C_{0}$, the $K$-types $\delta$, and the orbit types $O_{0}$ of spaces

$$
L_{C_{0}}^{2}\left(G / \Gamma ; \delta, O_{0} ;\{G\}\right) \text {. }
$$

Our objective is to prove that $L_{G / \Gamma}^{\text {dis }}(\alpha)$ is trace class for every $K$-finite $\alpha \in C_{c}^{\infty}(G)$. Because the sum over $C_{0}$ is finite, $C_{0}$ can be fixed, as can $\delta$, since Corollary 2.3 allows us to respond positively provided it can be shown that the restriction of

$$
\overline{\left(L_{G / \Gamma}\right)_{\infty}\left(1+\Delta^{N_{\delta}}\right)}{ }^{-1} L_{G / \Gamma}\left(\bar{\chi}_{\delta}\right)
$$

to

$$
\sum_{O_{0}} \bigoplus L_{C_{0}}^{2}\left(G / \Gamma ; \delta, O_{0} ;\{G\}\right)
$$

is Hilbert-Schmidt $\left(N_{\delta} \gg 0\right)$. 
To ensure placement in $L_{\text {res }}^{2}(G / \Gamma)$, we can and will suppose that $C_{0} \neq\{G\}$. Accordingly, given $O_{0}$, consider

$$
L_{\mathcal{C}_{0}}^{2}\left(G / \Gamma ; \delta, O_{0} ;\{G\}\right)
$$

i.e. (cf. TES, p. 339), consider

$$
\sum_{\mathfrak{E}} \bigoplus L_{\mathcal{C}_{0}}^{2}\left(G / \Gamma ; \delta, O_{0} ;\{G\} ; \mathfrak{E}\right)
$$

the summation $\sum_{\mathfrak{E}}$ being taken over the equivalence classes $\mathfrak{E}$ in $\mathfrak{X}\left(C_{0}\right)$. The discussion on p. 304 of TES implies, in turn, that for each $\mathfrak{E}, L_{\mathcal{C}_{0}}^{2}\left(G / \Gamma ; \delta, O_{0} ;\{G\} ; \mathfrak{E}\right)$ admits an orthonormal basis comprised of certain linear combinations

$$
f=\sum_{\mathfrak{X} \in \mathfrak{E}} C_{\mathfrak{X}} \cdot E\left(\mathfrak{X}: G\left|\{1\}: P_{i_{0}}\right| A_{i_{0}}: T_{i_{0}}: X\left(P_{i_{0}}, A_{i_{0}}\right): ?\right) .
$$

In this connection, we explicitly observe that

$$
E\left(\mathfrak{X}: G\left|\{1\}: P_{i_{0}}\right| A_{i_{0}}: T_{i_{0}}: X\left(P_{i_{0}}, A_{i_{0}}\right): ?\right)
$$

is an Eisenstein system function of a particular type.

Let $\omega_{0}$ be the Casimir operator per $C_{0}$. Then on

$$
L_{C_{0}}^{2}\left(G / \Gamma ; \delta, O_{0} ;\{G\}\right)
$$

we have $\square=\omega-c(\omega)$, where, in fact,

$$
c(\omega)=\chi\left(\omega_{0}\right)+c_{0} \quad\left(\chi \in O_{0}\right)
$$

$c_{0}$ being a scalar independent of $O_{0}$ (cf. TES, p. 346). Taking into account that all the normal translations $X\left(P_{i_{0}}, A_{i_{0}}\right)(\mathfrak{X} \in \mathfrak{E})$ have the same length,

$$
\square f=\left\|X\left(P_{i_{0}}, A_{i_{0}}\right)\right\|^{2} \cdot f .
$$

Consequently,

$$
\omega f=(\square+c(\omega)) f=\left(\chi\left(\omega_{0}\right)+c_{0}+\left\|X\left(P_{i_{0}}, A_{i_{0}}\right)\right\|^{2}\right) \cdot f
$$

or still

$$
\Delta f=\left(\omega_{K}-\omega\right) f=\left(\delta\left(\omega_{K}\right)-\chi\left(\omega_{0}\right)-c_{0}-\left\|X\left(P_{i_{0}}, A_{i_{0}}\right)\right\|^{2}\right) \cdot f .
$$

Therefore $f$ is an eigenfunction for $\Delta$. Furthermore, there is present a certain uniformity. Indeed, neither $c_{0}$ nor $\delta\left(\omega_{K}\right)$ depend on $O_{0}$, while the lengths of the normal translations are bounded above by $\left\|\rho_{i_{0}}\right\|$. So

LEMMA 3.1. There exists a constant $C_{\Delta}>0$, independent of $\mathrm{O}_{0}$, with the following property: If $\lambda$ is any eigenvalue of $\Delta$ in $L_{C_{0}}^{2}\left(G / \Gamma ; \delta, O_{0} ;\{G\}\right)$, then

$$
\left|\lambda+\chi\left(\omega_{0}\right)\right| \leq C_{\Delta} \quad\left(\chi \in O_{0}\right) .
$$

To make further progress, it will be necessary to assume that the reader is familiar with the theory of exponents, as spelled out in $\S 6$ of [12]. This said, maintaining the above supposition on $f$, we shall now find an estimate for $\#(\mathbf{E}(f, f))$. Fortunately, no real difficulty is present. Thus fix a $P_{0} \in C_{0}$. Then it is obvious that

$$
\#\left(E_{P_{0}}(f)\right) \leq \#\left(W\left(A_{0}\right)\right) \text {. }
$$


Let

$$
\left\{P_{i_{0} \mu_{0}}: 1 \leq i_{0} \leq r_{0}, 1 \leq \mu_{0} \leq r_{i_{0}}\right\}
$$

be a set of representatives for $\Gamma \backslash C_{0}$. Take a $\boldsymbol{\Lambda} \in \mathbf{E}(f, f)$. Then $\boldsymbol{\Lambda}$ is $P$-pure for some $P$ lying above some $P_{i_{0} \mu_{0}}$, say $\Lambda=\Lambda_{P}^{\prime}+\Lambda_{P}^{\prime \prime}$, where $\Lambda^{\prime} \in E_{P}(f)$ and $\Lambda^{\prime \prime} \in E_{P}(f)$. Observe that here both $\Lambda^{\prime}$ and $\Lambda^{\prime \prime}$ are real. Because $P$-purity is a function of the $\Gamma$-conjugacy class of $P$, on the basis of the Scholium to be found in the reference supra,

$$
\#\left(E_{P}(f)\right) \leq \#\left(W\left(A_{0}\right)\right) \cdot \sum_{i_{0}=1}^{r_{0}} r_{i_{0}} .
$$

Denoting by $N\left(C_{0}\right)$ the number of $\Gamma$-conjugacy classes of $\Gamma$-cuspidals dominating $C_{0}$, we have therefore proved

LEMMA 3.2. Suppose that

$$
f=\sum_{\mathfrak{X} \in \mathfrak{E}} C_{\mathfrak{X}} \cdot E\left(\mathfrak{X}: G\left|\{1\}: P_{i_{0}}\right| A_{i_{0}}: T_{i_{0}}: X\left(P_{i_{0}}, A_{i_{0}}\right): ?\right) .
$$

Then

$$
\#(\mathbf{E}(f, f)) \leq N\left(C_{0}\right) \cdot\left(\#\left(W\left(A_{0}\right)\right) \cdot \sum_{i_{0}=1}^{r_{0}} r_{i_{0}}\right)^{2}
$$

(Note. This estimate does not involve $O_{0}$ (nor, for that matter, $\left.\delta\right)$ )

4. Formulation of the RH and the GRH. The purpose of this section is to formulate the residue hypothesis $(\mathrm{RH})$ and the generalized residue hypothesis (GRH), the concomitant Main Conjecture (MC) being dealt with in $\S \S 7$ and 8, respectively. We shall, however, for heuristic and historical reasons, consider the $\Gamma$-rank-one case here.

The truth, in general, of the following statement is not known at the time of this writing.

MAIN CONJECTURE. The operator $L_{G / \Gamma}^{\mathrm{dis}}(\alpha)$ is trace class for every $K$-finite $\alpha$ in $C_{c}^{\infty}(G)$.

To establish the validity of the MC, it suffices, as we know (cf. $\S 3$ ), to fix $\delta$ and then produce an $N_{\delta}>0$ with the property that the restriction of

$$
\overline{\left(L_{G / \Gamma}\right)_{\infty}\left(1+\Delta^{N_{\delta}}\right)}{ }^{-1} L_{G / \Gamma}\left(\bar{\chi}_{\delta}\right)
$$

to

$$
\sum_{O_{0}} \bigoplus L_{c_{0}}^{2}\left(G / \Gamma ; \delta, O_{0} ;\{G\}\right)
$$

is Hilbert-Schmidt.

Again, as in $\S 3$, let

$$
f=\sum_{\mathfrak{X} \in \mathfrak{C}} C_{\mathfrak{X}} \cdot E\left(\mathfrak{X}: G\left|\{1\}: P_{i_{0}}\right| A_{i_{0}}: T_{i_{0}}: X\left(P_{i_{0}}, A_{i_{0}}\right): ?\right)
$$

be a typical eigenfunction for $\Delta$ in $L_{\mathcal{C}_{0}}^{2}\left(G / \Gamma ; \delta, O_{0} ;\{G\}\right)$. 
RESIDUE HYPOTHESIS. Suppose that there exists a constant $C_{\delta}>0$, independent of $\mathcal{O}_{0}$, such that $\forall \mathfrak{E}, \forall \mathfrak{X} \in \mathfrak{E}, \forall \lambda_{i_{0}} \in \sum_{P_{i_{0}}}^{0}\left(\mathfrak{g}, \mathfrak{a}_{i_{0}}\right)$,

$$
-\left(X\left(P_{i_{0}}, A_{i_{0}}\right), \lambda^{i_{0}}\right) \geq C_{\delta} .
$$

GENERALIZED RESIDUE HYPOTHESIS. Suppose that there exist constants $C_{\delta}>0, L_{\delta} \geq 0$, independent of $\mathcal{O}_{0}$, such that $\forall \mathfrak{E}, \forall \mathfrak{X} \in \mathfrak{E}, \forall \lambda_{i_{0}} \in \sum_{P_{i_{0}}}^{0}\left(\mathfrak{g}, \mathfrak{a}_{i_{0}}\right)$,

$$
-\left(X\left(P_{i_{0}}, A_{i_{0}}\right), \lambda^{i_{0}}\right) \geq C_{\delta} \cdot\left(1+\left|\chi\left(\omega_{0}\right)\right|\right)^{-L_{\delta}} \quad\left(\chi \in O_{0}\right) .
$$

(Note. As usual, $\lambda^{i_{0}}$ is the root dual to $\lambda_{i_{0}}$. To avoid any confusion, recall that Geom: III in TES implies that $\left(X\left(P_{i_{0}}, A_{i_{0}}\right), \lambda^{i_{0}}\right)$ is necessarily (strictly) negative.)

Because $L_{\delta}=0$ is admissible, obviously $\mathrm{RH} \Rightarrow \mathrm{GRH}$.

Henceforth, we shall assume the conditions of these hypotheses, our problem being, therefore, to see how they imply the MC.

Fix an orthonormal basis $\left\{f_{n}\right\}$ for

$$
\sum_{O_{0}} \bigoplus L_{C_{0}}^{2}\left(G / \Gamma ; \delta, O_{0} ;\{G\}\right)
$$

made up of $\Delta$-eigenfunctions, say $\Delta f_{n}=\lambda_{n} \cdot f_{n}$. Then

$$
\begin{aligned}
& \text { (a) } \lambda_{n} \geq 0 \\
& \text { (b) } \lambda_{n} \leq \lambda_{n+1} ; \\
& \text { (c) } \lambda_{n} \uparrow+\infty
\end{aligned}
$$

(Note. The last condition requires comment. It can happen that there are only finitely many $\lambda_{n}$ (e.g., if $\left.\operatorname{rank}(G / K)=1\right)$. Since what we want in this case is clear, we suppose, accordingly, that the number of $\lambda_{n}$ is infinite. But then, on the basis of a theorem of Borel and Garland (see TES, pp. 345-347), the $\lambda_{n}$ have no finite point of accumulation.)

Pick an element $\mathbf{H} \in \mathbf{a}_{Q}$ and put

$$
Q^{t}=Q^{\mathbf{H}-t \mathbf{H}_{\rho}} \quad(t \geq 0) .
$$

In terms of $t, \forall n$

$$
\left(Q^{t} f_{n}, f_{n}\right)=1-\sum_{\nu=1}^{N_{0}} p_{n, \nu}(t) e^{-s_{n, \nu} t} \quad\left(p_{n, \nu} \in \mathbf{R}[t]\right)
$$

where $1=\left(f_{n}, f_{n}\right)$. Furthermore, the $s_{n, \nu}$ are real, being certain "sums" of pairs of constant term exponents of $f_{n}$, thus are bounded above by $2\left\|\rho_{i_{0}}\right\|^{2}$ and are bounded below by

$$
C_{\delta}^{0} \cdot\left(1+\lambda_{n}\right)^{-L_{\delta}} \quad\left(C_{\delta}^{0}>0\right),
$$

as follows from Lemma 3.1, while $N_{0}$ is a certain absolute positive integer (cf. Lemma 3.2).

Owing to Proposition 2.4, $\forall t_{0}>0, \exists N\left(t_{0}\right)>0$ (depending on $\delta$ ) such that

$$
Q^{t_{0}} \circ \overline{\left(L_{G / \Gamma}\right)_{\infty}\left(1+\Delta^{N\left(t_{0}\right)}\right)}{ }^{-1} L_{G / \Gamma}\left(\bar{\chi}_{\delta}\right)
$$

is Hilbert-Schmidt. The relation

$$
Q^{t}=Q^{t} \circ Q^{t_{0}} \quad\left(0 \leq t \leq t_{0}\right)
$$


then implies that

$$
Q^{t} \circ{\overline{\left(L_{G / \Gamma}\right)_{\infty}\left(1+\Delta^{N\left(t_{0}\right)}\right)}}^{-1} L_{G / \Gamma}\left(\bar{\chi}_{\delta}\right)
$$

is likewise Hilbert-Schmidt. Since the inner product of

$$
Q^{t} \circ{\overline{\left(L_{G / \Gamma}\right)_{\infty}\left(1+\Delta^{N\left(t_{0}\right)}\right)}}^{-1} L_{G / \Gamma}\left(\bar{\chi}_{\delta}\right) f_{n}
$$

with itself is equal to

$$
\left(1+\lambda_{n}^{N\left(t_{0}\right)}\right)^{-2} \cdot\left(1-\sum_{\nu=1}^{N_{0}} p_{n, \nu}(t) e^{-s_{n, \nu} t}\right),
$$

we have, accordingly, that

$$
+\infty>\sum_{n}\left(1+\lambda_{n}^{N\left(t_{0}\right)}\right)^{-2} \cdot\left(1-\sum_{\nu=1}^{N_{0}} p_{n, \nu}(t) e^{-s_{n, \nu} t}\right),
$$

for all $t \in\left[0, t_{0}\right]$. In addition, it is clear that

$$
\sum_{\nu=1}^{N_{0}} p_{n, \nu}(t) e^{-s_{n, \nu} t} \in[0,1]
$$

Finally,

$$
0 \leq t^{\prime} \leq t^{\prime \prime} \Rightarrow \sum_{\nu=1}^{N_{0}} p_{n, \nu}\left(t^{\prime}\right) e^{-s_{n, \nu} t^{\prime}} \geq \sum_{\nu=1}^{N_{0}} p_{n, \nu}\left(t^{\prime \prime}\right) e^{-s_{n, \nu} t^{\prime \prime}}
$$

What must be done is to utilize this information to force

Objective. $\exists N_{\delta}>0$ st

$$
\sum_{n}\left(1+\lambda_{n}^{N_{\delta}}\right)^{-2}<+\infty
$$

Consequence. MC.

To illustrate, consider the simplest case of all, viz. when $\operatorname{rank}(\Gamma)=1$.

PROOF THAT $\mathrm{RH} \Rightarrow \mathrm{MC}(\operatorname{rank}(\Gamma)=1)$. In this situation,

$$
L_{\delta}=0, \quad N_{0}=1, \quad \operatorname{deg}\left(p_{n, \nu}\right)=0 .
$$

Take $t_{0}=1$. Then

$$
+\infty>\sum_{n}\left(1+\lambda_{n}^{N(1)}\right)^{-2} \cdot\left(1-c_{n} e^{-s_{n, \nu}}\right) .
$$

Because $c_{n} e^{-s_{n, \nu} t} \in[0,1], \forall t \geq 0$,

$$
t=0 \Rightarrow 0 \leq c_{n} \leq 1 \text {. }
$$

Thus

$$
1-c_{n} e^{-s_{n, \nu}} \geq 1-e^{-C_{\delta}^{0}}
$$

and so

$$
+\infty>\left(1-e^{-C_{\delta}^{0}}\right) \cdot \sum_{n}\left(1+\lambda_{n}^{N(1)}\right)^{-2}
$$

Our objective is therefore realized with the choice $N_{\delta}=N(1)$.

PROOF THAT GRH $\Rightarrow \mathrm{MC}(\operatorname{rank}(\Gamma)=1)$. In this situation,

$$
L_{\delta} \geq 0, \quad N_{0}=1, \quad \operatorname{deg}\left(p_{n, \nu}\right)=0,
$$


there being no loss of generality in assuming that actually $L_{\delta}>0$. Take $t_{0}=1$. Then

$$
\begin{aligned}
+\infty & >\sum_{n}\left(1+\lambda_{n}^{N(1)}\right)^{-2} \cdot\left(1-c_{n} e^{-s_{n, \nu}}\right) \\
& \geq \sum_{n}\left(1+\lambda_{n}^{N(1)}\right)^{-2} \cdot\left(1-e^{-s_{n, \nu}}\right) \\
& \geq \sum_{n}\left(1+\lambda_{n}^{N(1)}\right)^{-2} \cdot\left(1-e^{-C_{\delta}^{0} \cdot\left(1+\lambda_{n}\right)^{-L_{\delta}}}\right) .
\end{aligned}
$$

Noting that

$$
\lim _{n \rightarrow \infty} \frac{\left(1+\lambda_{n}\right)^{N}}{\left(1+\lambda_{n}^{N}\right)}=1
$$

we have

$$
\begin{aligned}
\lim _{n \rightarrow \infty} & \frac{\left(1+\lambda_{n}^{N(1)}\right)^{-2} \cdot\left(1-e^{-C_{\delta}^{0} \cdot\left(1+\lambda_{n}\right)^{-L_{\delta}}}\right)}{\left(1+\lambda_{n}^{N(1)+L_{\delta} / 2}\right)^{-2}} \\
& =\lim _{n \rightarrow \infty} \frac{\left(1+\lambda_{n}\right)^{-2 N(1)} \cdot\left(1-e^{-C_{\delta}^{0} \cdot\left(1+\lambda_{n}\right)^{-L_{\delta}}}\right)}{\left(1+\lambda_{n}\right)^{-2 N(1)-L_{\delta}}} \\
& =\lim _{n \rightarrow \infty} \frac{\left(1-e^{-C_{\delta}^{0} \cdot\left(1+\lambda_{n}\right)^{-L_{\delta}}}\right)}{\left(1+\lambda_{n}\right)^{-L_{\delta}}}=C_{\delta}^{0} .
\end{aligned}
$$

So, by limit comparison,

$$
+\infty>\sum_{n}\left(1+\lambda_{n}^{N(1)+L_{6} / 2}\right)^{-2} .
$$

Our objective is therefore realized with the choice $N_{\delta}=N(1)+\left[L_{\delta} / 2\right]+1$.

5. Constant term polynomials. The purpose of this section is to prove that the degrees of the $p_{n, \nu}$ figuring in the expansion

$$
\left(Q^{t} f_{n}, f_{n}\right)=1-\sum_{\nu=1}^{N_{0}} p_{n, \nu}(t) e^{-s_{n, \nu} t}
$$

can be bounded uniformly in $n$ and $\nu$.

Let $f, g \in \mathfrak{A}(G / \Gamma)$. Then, per a $\Gamma$-cuspidal $P(=M \cdot A \cdot N)$, we can write

$$
\left\{\begin{array}{l}
f_{P}(? m a)=\sum_{i} a^{\Lambda_{i}}\left(\sum_{k} p_{i k}(H) \Phi_{i k}(? m)\right) \\
g_{P}(? m a)=\sum_{j} a^{\Lambda_{j}}\left(\sum_{l} p_{j l}(H) \Phi_{j l}(? m)\right)
\end{array} \quad(H=\log a),\right.
$$

these decompositions being the customary "normal" forms of $f_{P}, g_{P}$ (see [12]). By definition, the $p_{i k}$ and $p_{j l}$ are the constant term polynomials of $f, g$ (with respect to $P$ ). This said, recall now that

$$
\left(Q^{\mathbf{H}} f, g\right)=\sum_{\mathbf{\Lambda} \in \mathbf{E}(f, g)} p_{\mathbf{\Lambda}}(\mathbf{H}) \cdot e^{\langle\mathbf{H}, \mathbf{\Lambda}\rangle}
$$

qua an exponential polynomial on $\mathfrak{a}_{Q}$. 
LEMMA 5.1. Suppose that the degrees of the constant term polynomials of $f, g$ are bounded by $d_{f}, d_{g}$, respectively. Then, $\forall \mathbf{\Lambda} \in \mathbf{E}(f, g)$,

$$
\operatorname{deg}\left(p_{\Lambda}\right) \leq d_{f}+d_{g}+\operatorname{rank}(\Gamma) .
$$

ProOF. Assuming, as we may, that the degree of $p_{\Lambda}$ is positive, select a "direction" $P=P_{m}^{\max }$ along which the degree of the corresponding derivative of $p_{\Lambda}$ is $\operatorname{deg}\left(p_{\mathbf{\Lambda}}\right)-1$. By hypothesis,

$$
\operatorname{deg}\left(p_{i k}\right) \leq d_{f} \quad \text { and } \operatorname{deg}\left(p_{j l}\right) \leq d_{g},
$$

thus, thanks to the transitivity of the constant term, the degrees of the constant term polynomials of $\Phi_{i k}, \Phi_{j l}$ are bounded by

$$
d_{f}-\operatorname{deg}\left(p_{i k}\right) \text { and } d_{g}-\operatorname{deg}\left(p_{j l}\right),
$$

respectively. Taking into account the differentiation principles to be found in $\S 5$ of $[\mathbf{1 2}]$, it follows by induction that

$$
\begin{aligned}
\operatorname{deg}\left(p_{\Lambda}\right)-1 \leq \max \{ & \operatorname{deg}\left(p_{i k}\right)+\operatorname{deg}\left(p_{j l}\right) \\
+ & {\left.\left[\left(d_{f}-\operatorname{deg}\left(p_{i k}\right)\right)+\left(d_{g}-\operatorname{deg}\left(p_{j l}\right)\right)+\operatorname{rank}(\Gamma)-1\right]\right\}, }
\end{aligned}
$$

i.e.,

or still

$$
\operatorname{deg}\left(p_{\Lambda}\right)-1 \leq d_{f}+d_{g}+\operatorname{rank}(\Gamma)-1
$$

as desired.

Because the $p_{n, \nu}$ are truncation polynomials, to bound their degrees uniformly, it suffices, therefore, to treat instead the constant term polynomials of the $f_{n}$ which, of course, are somewhat easier to handle.

Remarkably, it turns out that we can proceed in abstracto. Consider an irreducible unitary representation $U$ of $G$ occurring in $L_{\text {res }}^{2}(G / \Gamma)$, so

$$
H_{U} \hookrightarrow L_{\mathrm{res}}^{2}(G / \Gamma) \text {. }
$$

Denote by $H_{U}(K)$ the space of $K$-finite vectors in $H_{U}$. Then $H_{U}(K)$ is the algebraic direct sum over $\delta \in \hat{K}$ of the isotypic components $H_{U}(\delta)$. Let $f$ be an eigenvector for $\mathfrak{Z}(=$ center of $\mathfrak{G})$ in $H_{U}(\delta)$. Then $f \in \mathfrak{A}(G / \Gamma)$. Fix a $\Gamma$-cuspidal $P$. With $L=M \cdot A, \mathfrak{Z}_{L}=\mathfrak{Z}_{M} \otimes \mathfrak{Z}_{A}$ (cf. TES, p. 80). That being, call $\mu_{P}$ the canonical morphism $\mathfrak{Z} \rightarrow \mathfrak{Z}_{L}$. Then $\mathfrak{Z}_{L}$ is a free $\mathfrak{Z}$-module of finite rank $r_{L}$, say. It is well known that

$$
(Z f)_{P}=\mu_{P}(Z) f_{P} \quad(Z \in \mathfrak{Z})
$$

on $L$. On the other hand, $3 \cdot f=\mathbf{C} \cdot f$, and hence $\mu_{P}(\mathfrak{Z}) \cdot f_{P}=\mathbf{C} \cdot f_{P}$. Consequently, $\operatorname{dim}\left(\mathfrak{Z}_{L} \cdot f_{P}\right) \leq r_{L}$. Thanks to an observation of Wallach $[\mathbf{1 5}], \mathfrak{Z}_{L} \cdot f_{P}$ is contained in the Jacquet module $H_{U}(K) / \mathfrak{n} H_{U}(K)$. Now the latter can be expressed as a sum of generalized a-eigenspaces

$$
\left[H_{U}(K) / \mathfrak{n} H_{U}(K)\right]_{\Lambda} \quad(\Lambda: \mathfrak{a} \rightarrow \mathbf{C}),
$$

where, by definition,

$$
[\cdots]_{\Lambda}=\left\{a \in H_{U}(K):(\Lambda(H)+H)^{d_{\Lambda}} a=0(H \in \mathfrak{a})\right\} .
$$


Moreover, in view of the above remarks, it is clear that $d_{\Lambda} \leq r_{L}$. Finally, take a function $\phi \in\left(3_{L} \cdot f_{P}\right) \cap[\cdots]_{\Lambda}$. Let $\left\{p_{i}\right\}$ be a basis for the polynomials on a of degree $\leq d_{\Lambda}-1\left(<r_{L}\right)$. Then (cf. Langlands $[7$, p. 70])

$$
\phi(m a)=a^{\Lambda}\left(\sum_{i} p_{i}(H) \Phi_{i}(m)\right) \quad(H=\log a) .
$$

Here, needless to say, the $\Phi_{i} \in \mathfrak{A}\left(M / \Gamma_{M}\right)$. Since $\operatorname{deg}\left(p_{i}\right)<r_{L} \forall i$, from the fact that $f_{P} \in \mathfrak{Z}_{L} \cdot f_{P}$, we see at last that the polynomials per $f_{P}$ all have degree $<r_{L}$.

In particular,

PROPOSITION 5.2. The degrees of the $p_{n, \nu}$ are bounded uniformly in $n$ and $\nu$ by $N_{\Gamma} \equiv \max _{\Gamma \backslash C_{\Gamma}} r_{L}$.

(Note. $C_{\Gamma}$ is the set of $\Gamma$-cuspidal split parabolic subgroups of $G$.)

6. Laplace transformation of the differential equation. The purpose of this section is to assemble the data, the upshot being a reinterpretation of our problem as a question in analysis.

Suppose given positive integers $n_{0}$ and $N_{0}$, as well as:

(1) A sequence of real numbers $\left\{\lambda_{n}\right\}$ such that

(i) $\lambda_{n} \geq 0$

(ii) $\lambda_{n} \leq \lambda_{n+1}$;

(iii) $\lambda_{n} \uparrow+\infty$.

(2) A set of real numbers $\left\{s_{n, \nu}: 1 \leq \nu \leq N_{0}\right\}$, positive constants $A$ and $B$ and $L \geq 0$ such that $\forall n, \forall \nu, A /\left(1+\lambda_{n}\right)^{L} \leq s_{n, \nu} \leq B$.

(3) A set of polynomials $\left\{p_{n, \nu}: 1 \leq \nu \leq N_{0}\right\}$ in $\mathbf{R}[t]$ such that $\forall n, \forall \nu$,

(i) $\operatorname{deg}\left(p_{n, \nu}\right)<n_{0}$;

(ii) $\forall t \geq 0$,

$$
\sum_{\nu=1}^{N_{0}} p_{n, \nu}(t) e^{-s_{n, \nu} t} \in[0,1]
$$

(iii) $\forall t^{\prime \prime} \geq t^{\prime} \geq 0$,

$$
\sum_{\nu=1}^{N_{0}} p_{n, \nu}\left(t^{\prime}\right) e^{-s_{n, \nu} t^{\prime}} \geq \sum_{\nu=1}^{N_{0}} p_{n, \nu}\left(t^{\prime \prime}\right) e^{-s_{n, \nu} t^{\prime \prime}}
$$

Let (1), (2), (3) be tied together by

(*) $\forall t_{0}>0, \exists N\left(t_{0}\right)>0$ st $\forall t \in\left[0, t_{0}\right]$,

$$
+\infty>\sum_{n}\left(1+\lambda_{n}^{N\left(t_{0}\right)}\right)^{-2} \cdot\left(1-\sum_{\nu=1}^{N_{0}} p_{n, \nu}(t) e^{-s_{n, \nu} t}\right) .
$$

(Note. The residue hypotheses provide, of course, data of the above type.)

The claim then is $\exists N>0$ st $\sum_{n}\left(1+\lambda_{n}^{N}\right)^{-2}<+\infty$.

The proof of the claim is best divided into two cases: $L=0(\S 7), L>0(\S 8)$. Let us consider here the features common to both. 
LEMMA 6.1. The number of real zeros of $\sum_{\nu=1}^{N_{0}} p_{\nu}(t) e^{-s_{\nu} t}$ is bounded by

$$
\left[\sum_{\nu=1}^{N_{0}}\left(1+\operatorname{deg}\left(p_{\nu}\right)\right)\right]-1
$$

PROOF. We shall proceed by induction on

$$
\left[\sum_{\nu=1}^{N_{0}}\left(1+\operatorname{deg}\left(p_{\nu}\right)\right)\right]-1
$$

The initial step, namely when $N_{0}=1, \operatorname{deg}\left(p_{0}\right)=0$, is clear. Turning to the induction step, multiply the function through by $e^{s_{N_{0}} t}$ and differentiate. Then, for the derivative, the value of

$$
\left[\sum_{\nu=1}^{N_{0}}\left(1+\operatorname{deg}\left(p_{\nu}\right)\right)\right]-1
$$

is reduced by one. Owing to Rolle's lemma, the number of zeros of the derivative is $\geq$ the number of zeros of the original -1 . On the other hand, the number of zeros of the derivative is

$$
\leq\left[\sum_{\nu=1}^{N_{0}}\left(1+\operatorname{deg}\left(p_{\nu}\right)\right)\right]-1-1
$$

by induction. Hence the lemma.

In passing, observe that

$$
\left[\sum_{\nu=1}^{N_{0}}\left(1+\operatorname{deg}\left(p_{n, \nu}\right)\right)\right]-1 \leq n_{0} N_{0}-1 .
$$

The exponential polynomial

$$
\sum_{\nu=1}^{N_{0}} p_{n, \nu}(t) e^{-s_{n, \nu} t}
$$

is a solution to the linear differential equation

$$
D_{t}: \prod_{\nu=1}^{N_{0}}\left(\frac{d}{d t}+s_{n, \nu}\right)^{n_{0}} \text {. }
$$

Put

$$
\phi_{n}(t)=\sum_{\nu=1}^{N_{0}} p_{n, \nu}(t) e^{-s_{n, \nu} t}
$$

and, to be specific,

$$
p_{n, \nu}(t)=\sum_{i=0}^{n_{0}-1} a_{i}(n, \nu) t^{i}
$$

Then the Laplace transform $\mathcal{L}\left(\phi_{n}\right)$ of $\phi_{n}$ is equal to

$$
\left.\mathcal{L}\left(\phi_{n}\right)\right|_{s}=\sum_{\nu=1}^{N_{0}} \frac{q_{n, \nu}(s)}{\left(s+s_{n, \nu}\right)^{n_{0}}},
$$


where

$$
q_{n, \nu}(s)=\sum_{i=0}^{n_{0}-1} i ! a_{i}(n, \nu)\left(s+s_{n, \nu}\right)^{n_{0}-i-1}
$$

Unraveling the partial fractions, we have

$$
\left.\mathcal{L}\left(\phi_{n}\right)\right|_{s}=P_{n}(s) / \prod_{\nu=1}^{N_{0}}\left(s+s_{n, \nu}\right)^{n_{0}},
$$

$P_{n}$ a polynomial of degree $<n_{0} N_{0}$. Thanks to Laplace inversion (and Cauchy's theorem), $\phi_{n}$ can be recovered by writing

$$
\phi_{n}(t)=\frac{1}{2 \pi \sqrt{-1}} \int_{\mathfrak{C}} P_{n}(s) e^{t s} / \prod_{\nu=1}^{N_{0}}\left(s+s_{n, \nu}\right)^{n_{0}} d s .
$$

Here, $\mathfrak{C}$ is a smooth, positively oriented contour that symmetrically encloses $[-B,-A]$ if $L=0,[-B, 0]$ if $L>0$, and, in the first case, passes through $(-A / 2,0)$ but lies to the left of the line $x=-A / 2$.

These points made, drop the index $n$ from the notation, it now being merely excess baggage. As before, let $s_{1}, \ldots, s_{N_{0}}$ be real numbers lying between $A$ and $B$ (if $L=0$ ) or 0 and $B$ (if $L>0$ ). Then a generic solution to the linear differential equation $D_{t}: \prod_{\nu=1}^{N_{0}}\left(d / d t+s_{\nu}\right)^{n_{0}}$ is, say,

$$
\phi(t)=\sum_{\nu=1}^{N_{0}} p_{\nu}(t) e^{-s_{\nu} t}
$$

where $\forall \nu, p_{\nu}(t) \in \mathbf{R}[t]\left(\operatorname{deg}\left(p_{\nu}\right)<n_{0}\right)$. In view of the remarks supra, there are associations

the bottom arrow sending $P$ to

$$
\phi \rightarrow \mathcal{L}(\phi) \rightarrow P
$$

$$
\frac{1}{2 \pi \sqrt{-1}} \int_{\mathfrak{C}} P(s) e^{t s} / \prod_{\nu=1}^{N_{0}}\left(s+s_{\nu}\right)^{n_{0}} d s
$$

The resulting maps set up a linear one-to-one correspondence between the solution space Sol $\mathrm{t}_{\mathrm{t}}$ of $D_{t}$ and $V_{n_{0} N_{0}}$, the real polynomials of degree $<n_{0} N_{0}$.

(Note. We allow, as is permissible, repetitions in the $s_{\nu}$. Because of this, diffculties arise if we try to employ the usual methods.)

7. Proof that $\mathbf{R H} \Rightarrow \mathbf{M C}$. The purpose of this section is to prove that $\mathrm{RH}$ implies MC. In so doing, we shall use the set-up of the preceding section $(L=0)$, the objective being to produce an $N>0$ for which

$$
\sum_{n}\left(1+\lambda_{n}^{N}\right)^{-2}<+\infty
$$

Let $A, B, n_{0}, N_{0}$ be as in $\S 6$. Fix points $0<t_{1}<\cdots<t_{n_{0} N_{0}}$. Suppose that

$$
\phi(t)=\sum_{\nu=1}^{N_{0}} p_{\nu}(t) e^{-s_{\nu} t}
$$


is any exponential polynomial such that

(i) $\operatorname{deg}\left(p_{\nu}\right)<n_{0} \forall \nu$;

(ii) $A \leq s_{\nu} \leq B \forall \nu$;

(iii) $\left|\phi\left(t_{i}\right)\right| \leq 1 \forall i=1, \ldots, n_{0} N_{0}$. Then we claim that

$$
|\phi(t)| \leq M \cdot e^{(-A / 2) t} \quad(t \geq 0),
$$

$M$ depending on the constants but not on $\phi$. Admitting this estimate momentarily, let us see how it can be applied.

ProOF THAT RH $\Rightarrow$ MC. Take $t_{i}=i\left(i=1, \ldots, n_{0} N_{0}\right)$. Pick a $t_{0} \gg 0$ so as to make

$$
M \cdot e^{(-A / 2) t_{0}}<1 / 2 .
$$

Bearing in mind assumption (*), we now have

$$
\begin{aligned}
+\infty & >\sum_{n}\left(1+\lambda_{n}^{N\left(t_{0}\right)}\right)^{-2} \cdot\left(1-\sum_{\nu=1}^{N_{0}} p_{n, \nu}\left(t_{0}\right) e^{-s_{n, \nu} t_{0}}\right) \\
& >\frac{1}{2} \cdot \sum_{n}\left(1+\lambda_{n}^{N\left(t_{0}\right)}\right)^{-2} .
\end{aligned}
$$

Our objective is therefore realized with the choice $N=N\left(t_{0}\right)$.

(Note. This argument makes very coarse use of the data.)

To prove our claim, given any set $\left\{s_{\nu}: 1 \leq \nu \leq N_{0}\right\}$ of $s_{\nu}$ subject to $A \leq s_{\nu} \leq B$ $\forall \nu$, define a linear map

$$
T\left(s_{1}, \ldots, s_{N_{0}}\right): V_{n_{0} N_{0}} \rightarrow \mathbf{R}^{n_{0} N_{0}}
$$

by assigning to $P$ the vector $\left(\phi\left(t_{1}\right), \ldots, \phi\left(t_{n_{0} N_{0}}\right)\right)$, where, of course, $P$ and $\phi$ are linked by the Laplace transform (see $\S 6$ ). Thanks to Lemma $6.1, T\left(s_{1}, \ldots, s_{N_{0}}\right.$ ) is injective, thus, by dimension, is surjective, i.e., is an isomorphism. Let $K$ be the subset of $V_{n_{0} N_{0}}$ formed by taking the union over all possible sets $\left\{s_{\nu}: 1 \leq \nu \leq N_{0}\right\}$ of the counterimages

$$
T\left(s_{1}, \ldots, s_{N_{0}}\right)^{-1}\left([-1,1]^{n_{0} N_{0}}\right) .
$$

Then $K$ is compact, being the continuous image of

$$
[A, B]^{N_{0}} \times[-1,1]^{n_{0} N_{0}}
$$

under the map

$$
\left(s_{1}, \ldots, s_{N_{0}}\right) \times\left(x_{1}, \ldots, x_{n_{0} N_{0}}\right) \rightarrow T\left(s_{1}, \ldots, s_{N_{0}}\right)^{-1}\left(x_{1}, \ldots, x_{n_{0} N_{0}}\right) .
$$

With $\mathfrak{C}$ the contour of $\S 6$, put

$$
M_{\mathfrak{C}}=\sup _{P \in K}\|P \mid \mathfrak{C}\|_{\infty}, \quad d_{\mathfrak{C}}=\operatorname{dis}(\mathfrak{C},[-B,-A]) .
$$

Consider, finally, a $\phi$ satisfying (i), (ii) and (iii) above. Then, for $t \geq 0$, we have

$$
\begin{aligned}
|\phi(t)| & =1 / 2 \pi\left|\int_{\mathfrak{C}} P(s) e^{t s} / \prod_{\nu=1}^{N_{0}}\left(s+s_{\nu}\right)^{n_{0}} d s\right| \\
& \leq 1 / 2 \pi \cdot M_{\mathfrak{C}} / d_{\mathfrak{C}}^{n_{0} N_{0}} \cdot \operatorname{len}(\mathfrak{C}) \cdot e^{(-A / 2) t} \\
& \leq M \cdot e^{(-A / 2) t}
\end{aligned}
$$

the sought-for estimate. 
8. Proof that GRH $\Rightarrow$ MC. The purpose of this section is to prove that GRH implies MC. As in the discussion of the preceding section, we shall again use the set-up of $\S 6$, the objective once more being to produce an $N>0$ for which

$$
\sum_{n}\left(1+\lambda_{n}^{N}\right)^{-2}<+\infty
$$

However, due to the fact that the $s_{n, \nu}$ can approach zero, the proof is more complicated.

Let $A, B, n_{0}, N_{0}$ be as in $\S 6$. Equip the space $V_{n_{0} N_{0}}$ with the norm defined by the rule $\|P\|_{\mathbb{C}}=\|P \mid \mathfrak{C}\|_{\infty}$. Then, associated with each set $\left\{s_{\nu}: 1 \leq \nu \leq N_{0}\right\}$ of $s_{\nu}$ subject to $0 \leq s_{\nu} \leq B \forall \nu$, there is an injective morphism of Banach spaces

$$
\phi\left(s_{1}, \ldots, s_{N_{0}}\right): V_{n_{0} N_{0}} \rightarrow L^{1}([0,1]),
$$

the prescription being

$$
P \mapsto(2 \pi \sqrt{-1})^{-1} \int_{\mathfrak{C}} P(s) e^{t s} / \prod_{\nu=1}^{N_{0}}\left(s+s_{\nu}\right)^{n_{0}} d s \quad(0 \leq t \leq 1) .
$$

LEMMA 8.1. There is a constant $C_{\mathfrak{C}}>0$ such that $\forall s_{1}, \ldots, s_{N_{0}}: 0 \leq s_{\nu} \leq B$,

$$
\|P\|_{\mathfrak{C}} \leq C_{\mathfrak{C}} \cdot\left\|\phi\left(s_{1}, \ldots, s_{N_{0}}\right) P\right\|_{1} .
$$

It will be simplest to cite a generality.

SUBLEMMA. Let $X$ and $Y$ be Banach spaces; let $C$ be a subset of the bounded linear transformations $X \rightarrow Y$ such that

(i) $\forall T \in C, T$ is injective;

(ii) $\forall T \in C, T(X)$ is closed;

(iii) $C$ is compact (in the operator norm topology). Then $\exists \varepsilon>0$ st $\forall T \in C$ :

$$
\|T(x)\| \geq \varepsilon\|x\| \quad(x \in X) .
$$

ProOF. Owing to the open mapping theorem, $\forall T \in C, \exists \varepsilon_{T}>0$ st

$$
\|T(x)\| \geq \varepsilon_{T}\|x\| \quad(x \in X) .
$$

If $T_{0} \in C$ is fixed and if $T \in C$ is variable but within $\varepsilon_{T_{0}} / 2$ of $T_{0}$ in the operator norm topology, then, with $\|x\|=1$,

$$
\|T(x)\| \geq\left\|T_{0}(x)\right\|-\left\|T(x)-T_{0}(x)\right\| \geq \varepsilon_{T_{0}} / 2,
$$

so, by linearity,

$$
\|T(x)\| \geq\left(\varepsilon_{T_{0}} / 2\right)\|x\| \quad(x \in X) .
$$

On the other hand, in view of compactness, $C$ can be covered by finitely many balls $B\left(T_{i}, \varepsilon_{T_{i}} / 2\right)(i=1, \ldots, n)$. It remains only to put $\varepsilon=\min _{i}\left(\varepsilon_{T_{i}} / 2\right)$ in order to complete the proof.

Suppose now that $\phi$ is a solution to

$$
D_{t}: \prod_{\nu=1}^{N_{0}}\left(\frac{d}{d t}+s_{\nu}\right)^{n_{0}}
$$


Write $\phi=\phi\left(s_{1}, \ldots, s_{N_{0}}\right) P$. Thanks to the lemma, $\|P\|_{\mathfrak{C}} \leq C_{\mathfrak{C}} \cdot\|\phi\|_{1}$. Let $d_{\mathfrak{C}}=$ $\operatorname{dis}(\mathfrak{C},[-B, 0])$. Then it follows that

$$
\begin{aligned}
t \in[0,1] & \Rightarrow\left|\phi^{\prime}(t)\right|=1 / 2 \pi \cdot\left|\int_{\mathfrak{C}} P(s) s e^{t s} / \prod_{\nu=1}^{N_{0}}\left(s+s_{\nu}\right)^{n_{0}} d s\right| \\
& \leq 1 / 2 \pi \cdot\|P\|_{\mathfrak{C}} \cdot \sup \left\{\left|s e^{t s}\right|: s \in \mathfrak{C}, 0 \leq t \leq 1\right\} / d_{\mathfrak{C}}^{n_{0} N_{0}} \cdot \operatorname{len}(\mathfrak{C}) \\
& \leq C_{0} \cdot\|\phi\|_{1},
\end{aligned}
$$

if $C_{0}=\cdots$. Needless to say, $C_{0}$ does not depend on $\phi$. Integrating, we therefore get

$$
\left\|\phi^{\prime}\right\|_{1}=\int_{0}^{1}\left|\phi^{\prime}(t)\right| d t \leq C_{0} \cdot\|\phi\|_{1} .
$$

But $\phi^{\prime}$ is also a solution to

$$
D_{t}: \prod_{\nu=1}^{N_{0}}\left(\frac{d}{d t}+s_{\nu}\right)^{n_{0}}
$$

Accordingly, by iteration, we find that $\forall n \geq 1,\left\|\phi^{(n)}\right\|_{1} \leq C_{0}^{n-1} \cdot\left\|\phi^{\prime}\right\|_{1}$. To exploit this, expand the product $\prod_{\nu=1}^{N_{0}}\left(d / d t+s_{\nu}\right)^{n_{0}}$ as a sum

$$
\sum_{i=0}^{n_{0} N_{0}} a_{i}\left(s_{1}, \ldots, s_{N_{0}}\right) \frac{d^{i}}{d t^{i}}
$$

The $a_{i}\left(s_{1}, \ldots, s_{N_{0}}\right)$ are continuous on $[0, B]^{N_{0}}$, hence are bounded there, say

$$
\left|a_{i}\left(s_{1}, \ldots, s_{N_{0}}\right)\right| \leq M \quad \forall i .
$$

Taking into account the identity

$$
\sum_{i=1}^{n_{0} N_{0}} a_{i}\left(s_{1}, \ldots, s_{N_{0}}\right) \phi^{(i)}=-a_{0}\left(s_{1}, \ldots, s_{N_{0}}\right) \phi,
$$

we finally obtain an $L^{1}$-estimate of the form

$$
\begin{aligned}
\left|a_{0}\left(s_{1}, \ldots, s_{N_{0}}\right)\right| \cdot\|\phi\|_{1} & \leq \sum_{i=1}^{n_{0} N_{0}}\left|a_{i}\left(s_{1}, \ldots, s_{N_{0}}\right)\right| \cdot\left\|\phi^{(i)}\right\|_{1} \\
& \leq\left(M \cdot \sum_{i=1}^{n_{0} N_{0}} C_{0}^{i-1}\right) \cdot\left\|\phi^{\prime}\right\|_{1} .
\end{aligned}
$$

In other words,

LEMMA 8.2. There is an $\varepsilon>0$ with the property that $\forall s_{1}, \ldots, s_{N_{0}}: 0 \leq s_{\nu} \leq B$,

$$
\prod_{\nu=1}^{N_{0}}\left(\frac{d}{d t}+s_{\nu}\right) \phi=0 \Rightarrow\left\|\phi^{\prime}\right\|_{1} \geq \varepsilon \cdot \prod_{\nu=1}^{N_{0}} s_{\nu}^{n_{0}} \cdot\|\phi\|_{1} \text {. }
$$

(In this connection, note that $a_{0}\left(s_{1}, \ldots, s_{\nu}\right)=\prod_{\nu=1}^{N_{0}} s_{\nu}^{n_{0}}$.) 
Having paved the way, we are at last in a position to give the

PROOF THAT GRH $\Rightarrow M C$. To begin with, assumption (2) implies that

so, by Lemma 8.2 , if

$$
\prod_{\nu=1}^{N_{0}} s_{n, \nu}^{n_{0}} \geq \frac{A^{n_{0} N_{0}}}{\left(1+\lambda_{n}\right)^{n_{0} N_{0} L}}
$$

then

$$
\phi_{n}(t)=\sum_{\nu=1}^{N_{0}} p_{n, \nu}(t) e^{-s_{n, \nu} t}
$$

$$
\left\|\phi_{n}^{\prime}\right\|_{1} \geq \varepsilon \cdot \frac{A^{n_{0} N_{0}}}{\left(1+\lambda_{n}\right)^{n_{0} N_{0} L}} \cdot\left\|\phi_{n}\right\|_{1} .
$$

Next, from assumption (*) (with $t_{0}=1$ ),

$$
\begin{aligned}
+\infty & >\sum_{n}\left(1+\lambda_{n}^{N(1)}\right)^{-2} \cdot\left(1-\phi_{n}(1)\right) \\
& =\sum_{n: \phi_{n}(1) \leq 1 / 2} \cdots+\sum_{n: \phi_{n}(1)>1 / 2} \cdots
\end{aligned}
$$

Obviously,

$$
\begin{aligned}
+\infty & >\sum_{n: \phi_{n}(1) \leq 1 / 2}\left(1+\lambda_{n}^{N(1)}\right)^{-2} \cdot\left(1-\phi_{n}(1)\right) \\
& \geq \frac{1}{2} \cdot \sum_{n: \phi_{n}(1) \leq 1 / 2}\left(1+\lambda_{n}^{N(1)}\right)^{-2}
\end{aligned}
$$

thus, a fortiori,

$$
+\infty>\sum_{n: \phi_{n}(1) \leq 1 / 2}\left(1+\lambda_{n}^{N(1)+n_{0} N_{0} L / 2}\right)^{-2} .
$$

As for the other sum

$$
\sum_{n: \phi_{n}(1)>1 / 2}\left(1+\lambda_{n}^{N(1)}\right)^{-2} \cdot\left(1-\phi_{n}(1)\right)
$$

recall that $\phi_{n}$ is monotone decreasing (cf. assumption (3)), hence

$$
\begin{aligned}
0 \leq t \leq 1 & \Rightarrow \phi_{n}(t)>\frac{1}{2} \Rightarrow\left\|\phi_{n}\right\|_{1}>\frac{1}{2} \\
& \Rightarrow\left\|\phi_{n}^{\prime}\right\|_{1} \geq \frac{\varepsilon}{2} \cdot \frac{A^{n_{0} N_{0}}}{\left(1+\lambda_{n}\right)^{n_{0} N_{0} L}} .
\end{aligned}
$$

Because $1 \geq \phi_{n}(0)$ (assumption (3) again), we have

$$
\begin{aligned}
1 & \geq \phi_{n}(0)=\phi_{n}(1)-\int_{0}^{1} \phi_{n}^{\prime}(t) d t=\phi_{n}(1)+\left\|\phi_{n}^{\prime}\right\|_{1} \\
& \Rightarrow 1-\phi_{n}(1) \geq\left\|\phi_{n}^{\prime}\right\|_{1} \geq \frac{\varepsilon}{2} \cdot \frac{A^{n_{0} N_{0}}}{\left(1+\lambda_{n}\right)^{n_{0} N_{0} L}} \\
& \Rightarrow+\infty>\sum_{n: \phi_{n}(1)>1 / 2}\left(1+\lambda_{n}^{N(1)}\right)^{-2} \cdot\left(1-\phi_{n}(1)\right) \\
& \geq \frac{\varepsilon A^{n_{0} N_{0}}}{2} \cdot \sum_{n: \phi_{n}(1)>1 / 2}\left(1+\lambda_{n}^{N(1)}\right)^{-2} \cdot\left(1+\lambda_{n}\right)^{-n_{0} N_{0} L} .
\end{aligned}
$$


Our objective is therefore realized with the choice $N=N(1)+\left(n_{0} N_{0} L / 2\right)$.

(Note. This argument makes very strong use of the data.)

9. Consequences of the hypotheses. The purpose of this section is merely to make a few simple observations as regards what one can say if the $\mathrm{RH}$ or the GRH is granted.

Of course, the main point is the MC: The operator $L_{G / \Gamma}^{\mathrm{dis}}(\alpha)$ is trace class for every $K$-finite $\alpha$ in $C_{c}^{\infty}(G)$. That being, introduce, as usual, Harish-Chandra's Schwartz space $C^{1}(G)$ of integrable rapidly decreasing functions. Then, using the theory of the parametrix, it is not difficult to prove that $L_{G / \Gamma}^{\text {dis }}(\alpha)$ is still of the trace class if only $\alpha$ is a $K$-finite function in $C^{1}(G)$.

Next, recall that with $\mathbf{H} \in \mathfrak{a}_{Q}$,

$$
\lim _{\mathbf{H} \rightarrow-\infty} Q^{\mathbf{H}}=\text { ID }
$$

in the strong operator topology. So, if $\tau$ is the trace norm, then, for any $K$-finite $\alpha \in C^{1}(G), \tau\left(\left(Q^{\mathbf{H}}-\mathrm{ID}\right) L_{G / \Gamma}^{\mathrm{dis}}(\alpha)\right)$ must tend to zero when $\mathbf{H} \rightarrow-\infty$. Therefore,

$$
\operatorname{tr}\left(L_{G / \Gamma}^{\text {dis }}(\alpha)\right)=\lim _{\mathbf{H} \rightarrow-\infty} \operatorname{tr}\left(Q^{\mathbf{H}} L_{G / \Gamma}^{\text {dis }}(\alpha)\right)
$$

In addition,

$$
\operatorname{tr}\left(L_{G / \Gamma}^{\mathrm{dis}}(\alpha)\right)=\int_{G / \Gamma} K_{\alpha}^{\mathrm{dis}}(x, x) d_{G}(x) .
$$

It is to be explicitly observed that we have not proved that the linear functional $\operatorname{tr}\left(L_{G / \Gamma}^{\text {dis }}(?)\right)$ on the $K$-finite functions in $C_{c}^{\infty}(G)$ is continuous in the topology of $C_{c}^{\infty}(G)$. But if it is, then, by an abstract argument (see Proposition 4.8 in [9]), it can be shown that $L_{G / \Gamma}^{\mathrm{dis}}(\alpha)$ is of the trace class for every $\alpha \in C_{c}^{\infty}(G)$, the assignment

$$
\alpha \mapsto \operatorname{tr}\left(L_{G / \Gamma}^{\operatorname{dis}}(\alpha)\right) \quad\left(\alpha \in C_{c}^{\infty}(G)\right)
$$

being a distribution. Incidentally, these conclusions can then be readily extended to all of $C^{1}(G)$, where now, in view of the closed graph theorem, the assignment

$$
\alpha \mapsto \operatorname{tr}\left(L_{G / \Gamma}^{\operatorname{dis}}(\alpha)\right) \quad\left(\alpha \in C^{1}(G)\right)
$$

is continuous in the topology of $C^{1}(G)$.

10. Potpourri. The purpose of this section is to discuss, for the most part informally, the trace class question on $L_{\text {dis }}^{2}(G / \Gamma)$.

Write

$$
L_{\text {dis }}^{2}(G / \Gamma)=L_{\text {cus }}^{2}(G / \Gamma) \oplus L_{\text {res }}^{2}(G / \Gamma) \text {. }
$$

Then (see TES, p. 355), $\forall \alpha \in C^{1}(G)$, the operator $L_{G / \Gamma}^{\text {cus }}(\alpha)$ is trace class, the assignment

$$
\alpha \mapsto \operatorname{tr}\left(L_{G / \Gamma}^{\text {cus }}(\alpha)\right) \quad\left(\alpha \in C^{1}(G)\right)
$$

being, moreover, continuous in the topology of $C^{1}(G)$.

(Note. No $K$-finiteness requirement on $\alpha$ is needed for the validity of this statement.)

Ideally, one would like to be able to say that the assertion remains true when "cus" is replaced by "res". Unfortunately, the only situation of any generality in 
which something can definitely be said is the special case when $\operatorname{rank}(G / K)=1$, since then, for essentially trivial reasons, $L_{G / \Gamma}^{\text {res }}(\alpha)$ is trace class for all $K$-finite elements $\alpha$ in $C^{1}(G)$ (cf. [16]). Here, as will be recalled, the point is that given a $K$-type $\delta$, due to the compactness of $M$ only finitely many $M$-types can appear qua contituents of $\delta \mid M$.

(Note. Apart from certain exceptions, e.g., $G=\mathbf{S L}(2, \mathbf{R})$, it is unknown whether $K$-finiteness is necessary or not.)

If one considers the next simplest case, $\operatorname{viz} \cdot \operatorname{rank}(\Gamma)=1$ (but $\operatorname{rank}(G / K)$ arbitrary), then $M$ need not be compact, so, for instance, with $\delta=$ the trivial $K$-type, infinitely many class one representations of $M$ can potentially intervene, each contributing finitely many poles in $[-|\rho|, 0[$, which, conceivably, can pile up at the origin, the primary conundrum.

(Note. In his famous report, Selberg $[13]$ discusses $L^{2}(K \backslash G / \Gamma), \operatorname{rank}(\Gamma)=1$. On p. 185 of that paper (lines 6-12), he seems to suggest that he can, in fact, prove trace class on the full discrete spectrum. No proof has ever appeared.)

How is one to establish the traceability of $L_{G / \Gamma}^{\text {res }}(\alpha)$ ? For guidance, let us again look at the cuspidal case. The standard "soft" argument (cf. TES supra) seems to be of little use. Another approach has been employed by Donnelly $[4,5]$. The procedure is this. Fix a $K$-type $\delta$ which, in view of the usual bundle business, might just as well be taken trivial. Let $N_{\text {cus }}(\lambda)$ be the distribution function for the eigenvalues $\lambda_{n}$ of $\Delta$ on the cuspidal spectrum, i.e.,

$$
N_{\text {cus }}(\lambda)=\#\left(\left\{\lambda_{n}: \lambda_{n} \leq \lambda\right\}\right) .
$$

Put a $d=\operatorname{dim}(G / K)$. Then Donnelly has proved that

$$
\varlimsup_{\lambda \rightarrow \infty}\left(N_{\text {cus }}(\lambda) / \lambda^{d}\right) \leq C_{d} \cdot \operatorname{vol}(K \backslash G / \Gamma),
$$

$C_{d}$ a certain pcsitive constant depending only on $d$. This estimate leads at once to trace class. Thus, if $S_{N}=\left\{n: N \leq \lambda_{n}<N+1\right\}$, then

$$
\#\left(S_{N}\right) \leq C \cdot(N+1)^{d} \quad(\exists C>0)
$$

from which

$$
\begin{aligned}
\sum_{n} \frac{1}{\left(1+\lambda_{n}\right)^{d+2}} & =\sum_{N} \sum_{n \in S_{N}} \frac{1}{\left(1+\lambda_{n}\right)^{d+2}} \leq \sum_{N} \frac{\#\left(S_{N}\right)}{(N+1)^{d+2}} \\
& \leq C \cdot \sum_{N} \frac{(N+1)^{d}}{(N+1)^{d+2}} \leq C \cdot \sum_{N} \frac{1}{(N+1)^{2}}<+\infty .
\end{aligned}
$$

In reality, it is not so much the estimate which is of interest here as the methods used in getting it (cf. infra). Donnelly's procedure is based on general PDE considerations, coupled with the heat kernel method and uses very little else. There is, however, a serious obstacle in carrying over these results to the residual spectrum: What are the boundary conditions to be imposed? Cuspidality is easily reflected:

$$
\int_{N / N \cap \Gamma} ?=0
$$

a global $L^{2}$-condition. Regrettably, we see no obvious analogue for residuality, Harish-Chandra's theory of the weak constant term being applicable to automorphic forms only. 
(Note. A $\varliminf$ estimate for $N_{\text {cus }}(\lambda)$ is not known in general, even for $\mathbf{S L}(2, \mathbf{R})$, except, of course, for $\Gamma$ satisfying supplementary conditions of a particular kind (Venkov).)

REMARK. It is easy to see that

$$
\lambda_{n} \geq C_{1} \cdot n^{1 / t} \quad\left(\exists C_{1}>0\right)
$$

iff

$$
N_{\text {cus }}(\lambda) \leq C_{2} \cdot \lambda^{t} \quad\left(\exists C_{2}>0\right) .
$$

That being, the existence of an estimate of the form

$$
N_{\text {cus }}(\lambda) \leq C \cdot \lambda^{T} \quad(\exists C>0)
$$

for some $T>0$ is a simple consequence of the existence of the trace on $L_{\text {cus }}^{2}(G / \Gamma)$. Indeed, as is known (cf. [16]),

$$
\sum_{n} \frac{1}{\left(1+\lambda_{n}^{T}\right)}<+\infty \quad(\exists T \gg 0)
$$

So, Donnelly sharpens the $T$, itself of interest, but it is the way he does this which to us is the point.

Naturally, one cannot rule out further insights into the nature of the discrete spectrum from "external" developments. Spectral analysis on a complete Riemannian manifold, under suitable restrictions on the curvatures, is an active research topic, the journal literature growing yearly by leaps and bounds (cf. [14]). Nevertheless, our feeling is that these generalities are unlikely to bear fruit, basically because of their lack of specificity. To illustrate, a systematic treatment of the heat kernel on complete Riemannian manifolds may be found in [2 and 3] (see [6] for some applications), but these results seem to be of little use in resolving the trace class dilemma. Even when the class of manifolds is cut down to reflect the presence of "cusps" (as has been done by Müller in his elegant account [8]), the same old problems remain.

(Note. We have not relied on the heat kernel in our discussion since a certain degree of caution must be exercised in so doing. Consider the following example: Take $G=\mathbf{T}$ (the circle group) and let $U$ be the unitary representation of $G$ determined by the prescription $\bigoplus_{n \geq 0}\left[e^{\sqrt{n}}\right] \cdot e^{\sqrt{-1} n \pi}$.)

Can anything sensible be said at all? Let us look in more detail at the $\Gamma$-rank 1 situation.

Fix a $K$-type $\delta$. Let $O_{1}, O_{2}, \ldots$ be an enumeration of the orbit types reflecting the requirement that

$$
\left|\chi_{1}\left(\omega_{0}\right)\right| \leq\left|\chi_{2}\left(\omega_{0}\right)\right| \leq \cdots \quad\left(\chi_{1} \in O_{1}, \chi_{2} \in O_{2}, \ldots\right) .
$$

Attached to each orbit type $O_{i}$ is a finite set of eigenvalues $\lambda_{i, \iota}\left(\iota=1, \ldots, n_{i}\right)$, where

$$
\lambda_{i, \iota}=\left(\delta\left(\omega_{K}\right)-\chi_{i}\left(\omega_{0}\right)-c_{0}-\left\|X_{i, \iota}\left(P_{i_{0}}, A_{i_{0}}\right)\right\|^{2}\right) \quad\left(\chi_{i} \in O_{i}\right) .
$$

Pretending that $\rho$ does not exist, we shall write

$$
\sigma_{i, \iota}=\left\|X_{i, \iota}\left(P_{i_{0}}, A_{i_{0}}\right)\right\| \text {. }
$$

Then, with $f_{i, \iota} \leftrightarrow \lambda_{i, \iota}$,

$$
\left(Q^{t} f_{i, \iota}, f_{i, \iota}\right)=1-c_{i, \iota} e^{-2 \sigma_{i, \iota} t} \quad(t \geq 0) .
$$


Here, $c_{i, \iota}=\left\|\boldsymbol{\Phi}_{i, \iota}\right\|^{2} / 2 \sigma_{i, \iota}, \boldsymbol{\Phi}_{i, \iota}$ a certain element in $\mathcal{E}\left(\delta, O_{i}\right)$ depending on the initial selection of $\mathbf{H}$.

The notation set up, from the proof that GRH $\Rightarrow M C$, we know that $\forall L>0$, $\exists N_{L}>0$ st

$$
\sum_{i, \iota}\left(1+\lambda_{i, \iota}^{N_{L}}\right)^{-2}<+\infty,
$$

the indices of summation running through the $i, \iota$ having the property that

$$
\sigma_{i, \iota} \geq 1 / L \cdot\left(1+\left|\chi_{i}\left(\omega_{0}\right)\right|^{L}\right)^{-1} \text {. }
$$

Now deny trace class. Then, on the basis of what has been said earlier, $\forall L>0$, $\forall \varepsilon>0, \forall N>0$, the sum $\sum_{i, \iota}\left(1+\lambda_{i, \iota}^{N}\right)^{-2}$ over the $i, \iota$ for which

$$
\left.\left.\sigma_{i, \iota}<1 / L \cdot\left(1+\left|\chi_{i}\left(\omega_{0}\right)\right|^{L}\right)^{-1}, \quad c_{i, \iota} \in\right] 1-\varepsilon, 1\right]
$$

must diverge! Our intention will be to explore the plausibility of this conclusion.

To begin with, given $L$ and $\varepsilon$, denote by $S_{i}(L, \varepsilon)$ the set of all $\iota$ such that

$$
\left.\left.\sigma_{i, \iota}<1 / L \cdot\left(1+\left|\chi_{i}\left(\omega_{0}\right)\right|^{L}\right)^{-1}, \quad c_{i, \iota} \in\right] 1-\varepsilon, 1\right] \text {. }
$$

Then $\forall N>0$

$$
\sum_{i} \sum_{\iota \in S_{i}(L, \varepsilon)}\left(1+\lambda_{i, \iota}^{N}\right)^{-2}=+\infty .
$$

On the other hand, thanks to traceability on $L^{2}\left(M / \Gamma_{M}\right)$,

$$
\sum_{i} \operatorname{dim}\left(\varepsilon\left(\delta, O_{i}\right)\right) \cdot\left(1+\left|\chi_{i}\left(\omega_{0}\right)\right|^{N_{M}}\right)^{-2}<+\infty \quad\left(N_{M} \gg 0\right) .
$$

So, $\forall N>0$ (cf. Lemma 3.1),

$$
\sum_{i} \sum_{\iota \in S_{i}(L, \varepsilon)}\left(1+\left|\chi_{i}\left(\omega_{0}\right)\right|^{N_{M}+N}\right)^{-2}=+\infty
$$

In other words:

$$
\#\left(S_{i}(L, \varepsilon)\right) /\left(1+\left|\chi_{i}\left(\omega_{0}\right)\right|^{N}\right)
$$

is unbounded for all positive $N$ meaning, therefore, that "anomalous exponents" exist in abundance.

If $\iota \in S_{i}(L, \varepsilon)$, then

$$
\sqrt{2(1-\varepsilon) \sigma_{i, \iota}} \leq\left\|\Phi_{i, \iota}\right\| \leq \sqrt{2 \sigma_{i, \iota}}
$$

Accordingly,

$$
\begin{aligned}
\left(Q^{0} f_{i, \iota}, f_{i, \iota}\right) & =\left(Q^{0} f_{i, \iota}, Q^{0} f_{i, \iota}\right)=1-c_{i, \iota} \\
& =1-\left(\left\|\Phi_{i, \iota}\right\|^{2} / 2 \sigma_{i, \iota}\right) \leq \varepsilon .
\end{aligned}
$$

Thus, $\forall \iota \neq \iota^{\prime} \in S_{i}(L, \varepsilon)$,

$$
\left|\left(Q^{0} f_{i, \iota}, Q^{0} f_{i, \iota^{\prime}}\right)\right| \leq\left\|Q^{0} f_{i, \iota}\right\| \cdot\left\|Q^{0} f_{i, \iota^{\prime}}\right\| \leq \varepsilon .
$$

But also

$$
\begin{aligned}
\left|\left(Q^{0} f_{i, \iota}, Q^{0} f_{i, \iota^{\prime}}\right)\right| & =\left|\left(f_{i, \iota}, f_{i, \iota^{\prime}}\right)-\frac{1}{\sigma_{i, \iota}+\sigma_{i, \iota^{\prime}}} \cdot\left(\boldsymbol{\Phi}_{i, \iota}, \boldsymbol{\Phi}_{i, \iota^{\prime}}\right)\right| \\
& =\frac{1}{\sigma_{i, \iota}+\sigma_{i, \iota^{\prime}}} \cdot\left|\left(\boldsymbol{\Phi}_{i, \iota}, \boldsymbol{\Phi}_{i, \iota^{\prime}}\right)\right| \\
& \geq \frac{2(1-\varepsilon) \sqrt{\sigma_{i, \iota} \sigma_{i, \iota^{\prime}}}}{\sigma_{i, \iota}+\sigma_{i, \iota^{\prime}}} \cdot\left|\cos \left(\theta_{i}\left(\iota, \iota^{\prime}\right)\right)\right|
\end{aligned}
$$


$\boldsymbol{\theta}_{i}\left(\iota, \iota^{\prime}\right)$ the angle between $\boldsymbol{\Phi}_{i, \iota}$ and $\boldsymbol{\Phi}_{i, \iota^{\prime}}$ in $\mathcal{E}\left(\delta, O_{i}\right)$. Combining these facts leads now to the inequality

$$
\begin{aligned}
\varepsilon & \geq\left|\left(Q^{0} f_{i, \iota}, Q^{0} f_{i, \iota^{\prime}}\right)\right| \\
& \geq \frac{2(1-\varepsilon) \sqrt{\sigma_{i, \iota} \sigma_{i, \iota^{\prime}}}}{\sigma_{i, \iota}+\sigma_{i, \iota^{\prime}}} \cdot\left|\cos \left(\theta_{i}\left(\iota, \iota^{\prime}\right)\right)\right| .
\end{aligned}
$$

(Note. Bear in mind that $\iota \neq \iota^{\prime}$.)

To go further, it will be necessary to draw upon the important estimation principles of Langlands [7, pp. 100-116] which, when unraveled and specialized, serve to guarantee the existence of a positive constant $C\left(O_{i}\right)$ such that

$$
\left\|Q^{0} f_{i, \iota}\right\| \leq C\left(O_{i}\right) \cdot\left\|\boldsymbol{\Phi}_{i, \iota}\right\|
$$

Since

it follows that

$$
\begin{aligned}
\left|\left(Q^{0} f_{i, \iota}, Q^{0} f_{i, \iota^{\prime}}\right)\right| & \leq\left\|Q^{0} f_{i, \iota}\right\| \\
& \leq C\left(O_{i}\right) \cdot\left\|\boldsymbol{\Phi}_{i, \iota}\right\| \leq C\left(O_{i}\right) \cdot \sqrt{2 \sigma_{i, \iota}}
\end{aligned}
$$

$$
C\left(O_{i}\right) \cdot \sqrt{2 \sigma_{i, \iota}} \geq \frac{2(1-\varepsilon) \sqrt{\sigma_{i, \iota} \sigma_{i, \iota^{\prime}}}}{\sigma_{i, \iota}+\sigma_{i, \iota^{\prime}}} \cdot\left|\cos \left(\theta_{i}\left(\iota, \iota^{\prime}\right)\right)\right|
$$

or still

$$
\frac{C\left(O_{i}\right)}{\sqrt{2}(1-\varepsilon)} \geq \frac{\sqrt{\sigma_{\iota, \iota^{\prime}}}}{\sigma_{i, \iota}+\sigma_{i, \iota^{\prime}}} \cdot\left|\cos \left(\theta_{i}\left(\iota, \iota^{\prime}\right)\right)\right| .
$$

To exploit this relation, a lemma will be needed.

LEMMA. Let $k \geq 1$. Then there exists an $\varepsilon>0$ with the following property: If $\left\{e_{1}, \ldots, e_{2 n+k}\right\}$ is any set of unit vectors in $\mathbf{R}^{n}$, then $\exists i \neq j$ such that $\left(e_{i}, e_{j}\right) \geq \varepsilon$.

(We shall omit the proof, it being elementary and, in any event, well known.)

Per $n$ and $k$, set $\varepsilon_{n}(k)=\sup \varepsilon, \varepsilon$ as in the Lemma. We then ask:

Question. Do there exist integers $l_{0}$ and $L_{0}$ and a constant $C_{0}>0$ such that $\varepsilon_{n}\left(n^{l_{0}}\right) \geq C_{0} / n^{L_{0}}$ ?

(It is apparent that one is faced here with a chapter in "packing" theory, as yet not completely written; $\mathrm{cf}$. infra.)

For the sake of argument, assume the affirmative. Put $d_{i}=\operatorname{dim}\left(\mathcal{E}\left(\delta, O_{i}\right)\right)$. Then

$$
\#\left(S_{i}(L, \varepsilon)\right)-2 d_{i} \geq d_{i}^{l_{0}}
$$

for infinitely many $i$, so, on this set of $i$,

$$
\varepsilon_{d_{i}}\left(\#\left(S_{i}(L, \varepsilon)\right)-2 d_{i}\right) \geq C_{0} / d_{i}^{L_{0}} .
$$

However, as has been noted earlier,

$$
\begin{gathered}
\sum_{i} d_{i} \cdot\left(1+\left|\chi_{i}\left(\omega_{0}\right)\right|^{N_{M}}\right)^{-2}<+\infty \\
\Rightarrow d_{i} \leq C_{M} \cdot\left(1+\left|\chi_{i}\left(\omega_{0}\right)\right|^{N_{M}}\right)^{2} \quad\left(\exists C_{M}>0\right) .
\end{gathered}
$$

All told, therefore, on an infinite set of $i$,

$C$ a positive constant.

$$
\varepsilon_{d_{i}}\left(\#\left(S_{i}(L, \varepsilon)\right)-2 d_{i}\right) \geq \frac{C}{\left(1+\left|\chi_{i}\left(\omega_{0}\right)\right|^{\left.2 L_{0} N_{M}\right)}\right.},
$$


Returning to the inequality

$$
\frac{C\left(O_{i}\right)}{\sqrt{2}(1-\varepsilon)} \geq \frac{\sqrt{\sigma_{i, \iota^{\prime}}}}{\sigma_{i, \iota}+\sigma_{i, \iota^{\prime}}} \cdot\left|\cos \left(\theta_{i}\left(\iota, \iota^{\prime}\right)\right)\right|
$$

and looking only at the $i$ for which the preceding remarks are applicable, choose, as is possible, a pair $\iota \neq \iota^{\prime}$ such that

$$
\left|\cos \left(\theta_{i}\left(\iota, \iota^{\prime}\right)\right)\right|=\cos \left(\theta_{i}\left(\iota, \iota^{\prime}\right)\right) \geq \varepsilon_{d_{i}}\left(\#\left(S_{i}(L, \varepsilon)\right)-2 d_{i}\right) .
$$

Because there is no loss of generality in assuming that $\sigma_{i, \iota^{\prime}} \geq \sigma_{i, \iota}$, we then have

$$
\begin{aligned}
\frac{C\left(O_{i}\right)}{\sqrt{2}(1-\varepsilon)} & \geq \frac{1}{2 \sqrt{\sigma_{i, \iota^{\prime}}}} \cdot\left|\cos \left(\theta_{i}\left(\iota, \iota^{\prime}\right)\right)\right| \\
& \geq \frac{1}{2 \sqrt{\sigma_{i, \iota^{\prime}}}} \cdot \varepsilon_{d_{i}}\left(\#\left(S_{i}(L, \varepsilon)\right)-2 d_{i}\right) \\
& \geq \frac{1}{2 \sqrt{\sigma_{i, \iota^{\prime}}}} \cdot \frac{C}{\left(1+\left|\chi_{i}\left(\omega_{0}\right)\right|^{\left.2 L_{0} N_{M}\right)}\right.} \\
& \geq \frac{C}{2} \cdot \frac{1}{\sqrt{\left(1 / L \cdot\left(1+\left|\chi_{i}\left(\omega_{0}\right)\right|^{L}\right)^{-1}\right)}} \frac{1}{\left(1+\left|\chi_{i}\left(\omega_{0}\right)\right|^{\left.2 L_{0} N_{M}\right)}\right.}
\end{aligned}
$$

i.e.,

$$
C\left(O_{i}\right) \geq \frac{C(1-\varepsilon)}{\sqrt{2}} \cdot \sqrt{L} \cdot\left(1+\left|\chi_{i}\left(\omega_{0}\right)\right|^{L}\right)^{1 / 2} \frac{1}{\left(1+\left|\chi_{i}\left(\omega_{0}\right)\right|^{\left.2 L_{0} N_{M}\right)}\right.} .
$$

But neither $L_{0}$ nor $N_{M}$ depend on $L$. Thus it follows that $C\left(O_{i}\right)$ blows up faster than any polynomial in $\left|\chi_{i}\left(\omega_{0}\right)\right|$, a quite unlikely inference.

To summarize. A possible approach to trace class on $L_{\mathrm{res}}^{2}(G / \Gamma)$ is through the question supra and a careful study of the growth of $C\left(O_{i}\right)$ vis-à-vis $\left|\chi_{i}\left(\omega_{0}\right)\right|$.

(Note. Actually, we are of the opinion that the conditions of the GRH are probably true. Needless to say, a proof of their validity would be very interesting.)

Adopting a geometric posture, let us try to convince ourselves that the answer to our question is "yes", in fact that even

$$
\varepsilon_{n}\left(n^{2}\right) \geq C_{0} / n \quad\left(\exists C_{0}>0\right) .
$$

The argument is suggested by a result of Coxeter (Proc. Sympos. Pure Math., vol. 7, Amer. Math. Soc., Providence, R.I., 1963, pp. 53-71), itself, sad to say, conjectural. Let an angle $\phi<\pi / 4$ be given. Call $N_{n}(\phi)$ the maximum number of nonoverlapping spherical caps of angular radius $\phi$ on the $n$-sphere $\mathbf{S}^{n-1}$. Then, employing a certain "intuitively obvious" geometric lemma which has never been established in all detail, Coxeter proves that $\forall M>1, \exists N_{M, \phi}$ st

$$
N_{n}(\phi) \leq M \cdot \frac{2^{1-n / 2} \sqrt{\pi \cos (2 \phi)} n^{3 / 2}}{e \sin ^{n-1}(\phi)} \quad\left(\forall n>N_{M, \phi}\right)
$$

We explicitly observe that this estimate cannot hold at $\phi=\pi / 4$ since then $\cos (2 \phi)=$ 0 while, as is known, $N_{n}(\pi / 4)=2 n$. It is not difficult to check that the constant $N_{M, \phi}$ can be chosen uniformly for $\phi$ in intervals of the form $\left.] \varepsilon, \pi / 4-\varepsilon\right]$, so we have

$$
N_{n}(\phi) \leq M_{\varepsilon} \cdot(\sqrt{\cos (2 \phi)} \sin (\phi)) \cdot n^{3 / 2} /(\sqrt{2} \sin (\phi))^{n}
$$


for all $n$ and for all $\phi$ in $] \varepsilon, \pi / 4-\varepsilon]$. Now throw away the trigonometric numerator. Then still

$$
N_{n}(\phi) \leq M_{\varepsilon} \cdot \frac{n^{3 / 2}}{(\sqrt{2} \sin (\phi))^{n}}
$$

on $] \varepsilon, \pi / 4-\varepsilon]$. But this inequality at least makes sense all the way up to $\pi / 4$. Accordingly, admit its validity on $] \varepsilon, \pi / 4$ ] (a proof would probably involve an examination of the finer properties of the Schläfi functions). Determine $\phi_{n}$ by the requirement that $\cos \left(2 \phi_{n}\right)=1 / n$. Then

$$
\begin{aligned}
N_{n}\left(\phi_{n}\right) & \leq M_{\varepsilon} \cdot 1 / \sqrt{(1-1 / n)^{n}} \cdot n^{3 / 2} \\
& \sim M_{\varepsilon} \cdot 1 / \sqrt{e^{-1}} \cdot n^{3 / 2},
\end{aligned}
$$

that is,

$$
N_{n}\left(\phi_{n}\right) \leq M_{0} \cdot n^{3 / 2} \quad\left(\exists M_{0} \gg 0\right) .
$$

Claim. $\varepsilon_{n}\left(1+\left[M_{0} \cdot n^{3 / 2}\right]\right) \geq 1 / n$.

To see this, simply observe that if we have $1+\left[M_{0} \cdot n^{3 / 2}\right]+2 n$ vectors, with no ordered pair having an associated angle $\leq 2 \phi_{n}$, then the corresponding spherical caps of angular radius $\phi_{n}$ are nonoverlapping, which cannot be. Finally, therefore,

$$
\varepsilon_{n}\left(n^{2}\right) \geq C_{0} / n \quad\left(\exists C_{0}>0\right) .
$$

Is it possible to make this discussion totally rigorous? Of course, one will somehow have to avoid the use of Coxeter's estimate, the point being to prove a priori that

$$
N_{n}\left(\phi_{n}\right) \leq M_{0} \cdot n^{3 / 2} \quad\left(\exists M_{0} \gg 0\right) .
$$

To do so, we shall make use of a result due to V. Levenstein (Soviet Math. Dokl. 20 (1979), 417-421), the thrust of which is to provide an interpolation to the left of $\pi / 4$. Precisely put, he shows that

$$
N_{n}(\phi) \leq \frac{n(1-\cos (2 \phi))(2+(n+1) \cos (2 \phi))}{1-n(\cos (2 \phi))^{2}}
$$

if

$$
0 \leq \cos (2 \phi) \leq 1 /(\sqrt{n+3}+1) .
$$

In passing, observe that this gives $N_{n}(\pi / 4) \leq 2 n$, whereas, as already mentioned, $N_{n}(\pi / 4)=2 n$. Supposing that $n \gg 0$ and keeping in mind that $\cos \left(2 \phi_{n}\right)=1 / n$, we can therefore say that

$$
N_{n}\left(\phi_{n}\right) \leq 3 n+1 \leq M_{0} \cdot n^{3 / 2} \quad\left(\exists M_{0} \gg 0\right)
$$

the desired inequality.

It remains to say a word or two about the $C\left(O_{i}\right)$. As we mentioned earlier, their existence is guaranteed by some results of Langlands [7] (see, in particular, Lemma 5.2). His method is to produce the $C\left(O_{i}\right)$ by a sequential argument, i.e., is a proof by contradiction, hence is implicitly nonconstructive and therein lies the rub. Because virtually all constructive estimates in the theory turn out to be of polynomial type in the parameters, we think that it is certainly reasonable to expect that

$$
C\left(\mathcal{O}_{i}\right) \leq C \cdot\left(1+\left|\chi_{i}\left(\omega_{0}\right)\right|\right)^{N} \quad(\exists C, N>0) .
$$


Granted this, our discussion then leads to trace class, i.e., to $\mathrm{MC}$ (when $\operatorname{rank}(\Gamma)=$ 1).

In conclusion, it seems appropriate to comment on the conditions for trace class which were given in [9]. On p. 49 of that work, we made the following assumption.

Assumption. Let $\alpha$ be a $K$-finite function in $C_{c}^{\infty}(G)$. Then

$$
\sum_{0} \operatorname{tr}\left(\phi_{\alpha}(O, t) \cdot \frac{\mathbf{c}_{\mathcal{O}}(t)-\mathbf{c}_{\mathcal{O}}(0)}{t}\right) \in L^{1}(-\varepsilon, \varepsilon) \text {. }
$$

The significance of this assumption was that it led to trace class on the discrete spectrum (and even somewhat more); cf. Proposition 8.8 in [9]. Moreover, on p. 51 of that work we wrote down a list of three conditions, each stronger than its predecessor, and each of which implied the assumption supra. Omitting the details (not trivial but not hard...), we want now to observe that each of these conditions can be weakened to allow polynomial growth in the parameters without altering the basic conclusion. More precisely, consider the following conditions:

(i) $\exists C>0, \exists M, N>0$ st $\forall 0$

$$
\int_{-\varepsilon(0)}^{\varepsilon(0)} \log |t| \cdot\left\|\mathbf{c}_{O}^{\prime}(t)\right\|_{O P} d t \leq C \cdot\left(1+\left|\chi\left(\omega_{0}\right)\right|\right)^{N},
$$

where

$$
\varepsilon(0)=1 /\left(1+\left|\chi\left(\omega_{0}\right)\right|\right)^{M} \quad(\chi \in 0) .
$$

(ii) $\exists C>0, \exists M, N>0$ st $\forall 0$

$$
\left\|\mathbf{c}_{O}^{\prime}(t)\right\|_{O P} \leq C \cdot\left(1+\left|\chi\left(\omega_{0}\right)\right|\right)^{N}
$$

for all real $t$ with

$$
|t| \leq 1 /\left(1+\left|\chi\left(\omega_{0}\right)\right|\right)^{M} \quad(\chi \in 0) .
$$

(iii) $\exists C>0, \exists M, N>0$ st $\forall 0$

$$
\left\|\mathrm{c}_{O}(t)\right\|_{O P} \leq C \cdot\left(1+\left|\chi\left(\omega_{0}\right)\right|\right)^{N}
$$

for all complex $t$ with

$$
|t| \leq 1 /\left(1+\left|\chi\left(\omega_{0}\right)\right|\right)^{M} \quad(\chi \in 0) .
$$

Then, (iii) $\Rightarrow($ ii) $\Rightarrow$ (i). Furthermore, (i) implies our assumption.

(Note. For (iii) to hold, the conditions of the GRH must necessarily be in force.)

ADDED IN PROOF. Recently, in a manuscript with the title Eigenvalue estimates for certain noncompact manifolds, $\mathrm{H}$. Donnelly has succeeded in verifying $\mathrm{MC}$ when $\operatorname{rank}(\Gamma)=1$. His proof is based on a refinement of the ideas in [4 and 5]. It seems very likely that, using the partition of $G / \Gamma$ established in $[10]$. Donnelly's method can be extended to cover the general case, thus proving MC for $\Gamma$ of any rank. On the other hand, MC itself seems to say nothing about RH or GRH.

\section{REFERENCES}

1. J. Arthur, The trace formula for reductive groups, Publ. Math. Univ. Paris VII, vol. 15, Paris, 1983, pp. 1-41.

2. J. Cheeger and S.-T. Yau, A lower bound for the heat kernel, Comm. Pure Appl. Math. 34 (1981), 465-480. 
3. S. Cheng, P. Li and S.-T. Yau, On the upper estimate of the heat kernel of a complete Riemannian manifold, Amer. J. Math. 103 (1981), 1021-1063.

4. H. Donnelly, On the point spectrum for finite volume symmetric spaces of negative curvature, Comm. Partial Differential Equations 6 (1981), 963-992.

5. 239-253.

6. H. Donnelly and P. Li, Lower bounds for the eigenvalues of negatively curved manifolds, Math. Z. 172 (1980), 29-40.

7. R. Langlands, On the functional equations satisfied by Eisenstein series, Lecture Notes in Math., vol. 544, Springer-Verlag, Berlin and New York, 1976, 1-337.

8. W. Müller, Spectral theory for Riemannian manifolds writh cusps and a related trace formula, Math. Nachr. 111 (1983), 197-288.

9. S. Osborne and G. Warner, The Selberg trace formula. I, Crelle's J. 324 (1981), 1-113.

10. __ The Selberg trace formula. II, Pacific J. Math. 106 (1983), 307-496.

11. _ The Selberg trace formula. III, Mem. Amer. Math. Soc. No. 44 (1983), 1-209.

12. __ The Selberg trace formula. IV, Lecture Notes in Math., vol. 1024, Springer-Verlag, Berlin and New York, 1983, pp. 112-263.

13. A. Selberg, Discontinuous groups and harmonic analysis, (Proc. Internat. Congr. of Math., Stockholm, 1962), Almquist \& Wiksells, Uppsala, 1963, pp. 177-189.

14. R. Strichartz, Analysis of the Laplacian on a complete Riemannian manifold, J. Funct. Anal. 52 (1983), pp. 48-79.

15. N. Wallach, On the constant term of a square integrable automorphic form, preprint.

16. G. Warner, Selberg's trace formula for nonuniform lattices: The $\mathbf{R}$-rank 1 case, Adv. in Math. Suppl. Stud., vol. 6, Academic Press, New York, 1979, pp. 1-142.

Department of Mathematics, UNiVERsity of Washington, Seattle, WashingTON 98195 